\title{
SOLID CARBONYL SULPHIDE (OCS) IN W33A
}

\author{
M. E. Palumbo \\ Istituto di Astronomia, Università di Catania, Viale A. Doria 6, 95125 Catania, Italy \\ A. G. G. M. TIELENS \\ NASA/Ames Research Center, MS 245-3, Moffett Field, CA 94035 \\ AND \\ A. T. Tok Unaga \\ Institute for Astronomy, University of Hawaii, 2680 Woodlawn Drive, Honolulu, HI 96822 \\ Received 1994 November 16; accepted 1995 March 6
}

\begin{abstract}
We present ground-based observations of the $2041 \mathrm{~cm}^{-1}(4.9 \mu \mathrm{m})$ absorption feature toward the deeply embedded protostar W33A.

We attribute this interstellar feature to solid carbonyl sulphide (OCS) embedded in icy grain mantles along the line of sight. We have made an extensive laboratory study of the infrared characteristics of OCS in various ice mixtures. Studies of the $2041 \mathrm{~cm}^{-1}$ band of solid OCS and solid mixtures containing OCS show that its shape and peak position are sensitive to the molecular environment of the OCS molecule. Mie scattering calculations show that the peak position and profile of the OCS band depend on the shape and size of the absorbing grain when the OCS concentration is larger than 0.05. For lower OCS concentrations, laboratorymeasured bulk spectra agree very well with the absorption spectra of small grains.

We have compared the observed feature with laboratory and theoretical spectra of astrophysically relevant mixtures. The best agreement is obtained for mixtures with traces of OCS in a methanol-rich matrix. This would suggest the presence of independent grain components and in particular of a minor fraction of methanol-rich icy grain mantles in which OCS is embedded. From the strength of the absorption feature we deduce a $\mathrm{OCS} / \mathrm{H}_{2} \mathrm{O}$ ratio, along the line of sight, of $4 \times 10^{-4}$.

Subject headings: infrared: ISM : lines and bands - ISM : clouds - ISM : individual (W33) -
\end{abstract}

ISM : molecules - line: identification - molecular processes

\section{INTRODUCTION}

Icy grain mantles are known to be an important component of dust in molecular clouds. Their presence is clearly indicated by IR absorption features, characteristic of frozen molecules, observed toward many obscured sources. In particular, many of the observed features have first been detected toward the deeply embedded young stellar source W33A and are generally more prominent in this source than in any other (Lacy et al. 1984; Tielens et al. 1984). These features are generally attributed to simple molecules such as $\mathrm{H}_{2} \mathrm{O}, \mathrm{CH}_{3} \mathrm{OH}$, and $\mathrm{CO}$ (Tielens \& Allamandola 1987b; Tielens 1989). Icy grain mantles are formed by accretion and reaction of gas phase species onto preexisting grain cores (Tielens \& Hagen 1982; d'Hendecourt, Allamandola, \& Greenberg 1985; Brown, Charnley, \& Millar 1988). UV and cosmic-ray processing of these icy mantles may lead to the formation of other, more complex, molecules (Geballe et al. 1985; Grim \& Greenberg 1987; Palumbo \& Strazzulla 1993) and eventually to the formation of a complex refractory organic residue which, unlike volatile icy mantles, will probably survive in the diffuse interstellar medium (Greenberg 1982; Baratta \& Strazzulla 1990).

In this paper we present an observational and laboratory study of the $2041 \mathrm{~cm}^{-1}$ carbonyl sulfide (OCS) band. The detection of an absorption feature at $2041 \mathrm{~cm}^{-1}(4.9 \mu \mathrm{m})$ has already been reported by Larson et al. (1985) and Geballe et al. (1985), and several carriers have been suggested. Based on laboratory experiments Geballe et al. proposed the identification with an unidentified sulfur-containing molecule, while Larson et al. suggested $\mathrm{C}_{3}, \mathrm{CN}$, and $\mathrm{CH}_{3} \mathrm{OH}$, and Grim et al.
(1991) suggested $\mathrm{CH}_{3} \mathrm{OH}$ and an unidentified absorber. Experimental studies of the $2041 \mathrm{~cm}^{-1}$ band of solid OCS (Hudgins et al. 1993; this work) show that its profile (shape, width, and peak position) strongly depends on the host molecules when it is mixed in with other species as well as on the temperature. Hence, comparisons of laboratory spectra with observations can provide information on the chemical and physical conditions of interstellar ices containing OCS. In this work we attribute the $2041 \mathrm{~cm}^{-1}(4.9 \mu \mathrm{m})$ feature observed toward W33A to solid OCS in grain mantles. In $\S 2$ we discuss the observational procedure. In $\S 3$ we present laboratory IR spectra of ice mixtures containing OCS. The effects of particle size and shape on the profile of absorption features and Mie scattering calculations are described in $\$ 4$. Then, laboratory and calculated spectra are compared with the observed feature and astrophysical implications are discussed in $\S 5$. The need for further observational and laboratory studies is pointed out in $\$ 6$.

\section{OBSERVATIONS AND DATA REDUCTION}

Spectra of W33A in the $4-5 \mu \mathrm{m}$ range were obtained using the Infrared Telescope Facility (IRTF) on Mauna Kea, HI, on 1988 July 21. The spectra were measured using the Cooled Grating Array Spectrometer (CGAS) a 32 element InSb detector, $\mathrm{LN}_{2}$ cooled (Tokunaga, Smith, \& Irwin 1987). The observations were made using an aperture of $2 " .7$ and a north-south chop of $15^{\prime \prime}$. Integration time was 4 minutes. The spectrometer was used in first order with grating " $B$ " to provide a resolving power of 1200 (i.e., $\Delta \lambda=0.004 \mu \mathrm{m} \sim 1.8 \mathrm{~cm}^{-1}$ ). The spectrum 



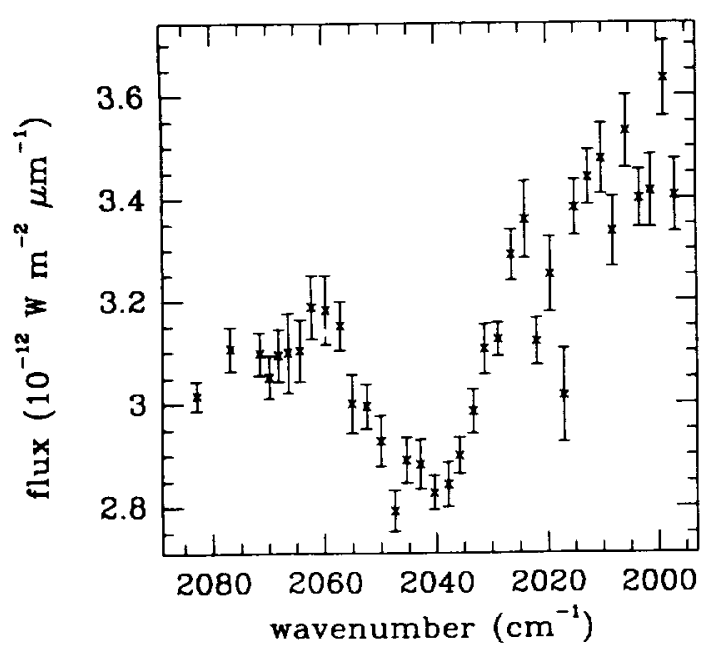

Fig. 1.-The $2041 \mathrm{~cm}^{-1}(4.9 \mu \mathrm{m})$ feature observed toward W33A. Noisy points around $2020 \mathrm{~cm}^{-1}$ do not define a real feature.

was Nyquist sampled. Calibration and correction for atmospheric absorption were accomplished by comparison with the nearby bright star BS 6561 (spectral type F0 IV) for which spectra were measured through similar air mass. Absolute wavelength calibration was provided by an argon discharge lamp included in CGAS.

In order to improve signal-to-noise ratio, we have binned the original data, replacing each set of three points by the weighted mean value. This degrades the resolving power to 400 . For comparison with laboratory data, a linear baseline will be adopted. This has been drawn between 2010 and 2060 $\mathrm{cm}^{-1}(4.97-4.85 \mu \mathrm{m})$ and has been defined using the mean value of the flux from six data points on both sides of the absorption feature. The observed feature (Fig. 1) peaks at 2041 $\mathrm{cm}^{-1}(4.9 \mu \mathrm{m})$, and its FWHM, in a linear intensity scale, is 22 $\mathrm{cm}^{-1}(0.0529 \mu \mathrm{m})$. Our spectrum agrees well in shape and peak position with the previous spectrum by Geballe et al. (1985).

\section{LABORATORY STUDIES OF OCS}

Laboratory experiments discussed in this paper have been performed at NASA/Ames or in the laboratory of experimental astrophysics at Catania (Italy). For a detailed description of the experimental apparata, see Allamandola, Sandford, \&
Valero (1988) and Strazzulla \& Baratta (1991), respectively. Our new experimental results are summarized in Table 1 and compared in Figure 2 to earlier studies by Hudgins et al. (1993).

OCS is a linear molecule with a permanent dipole moment of 0.7 debye which appears in the gas phase at room temperature. The IR spectrum of pure OCS ice at $10 \mathrm{~K}$ shows an intense absorption band, due to the $\mathrm{C}=\mathrm{O}$ stretch, at 2025 $\mathrm{cm}^{-1}$ (also referred to as the asymmetric stretch by Hudgins et al. 1993) with FWHM of $48 \mathrm{~cm}^{-1}$ measured on an intensity scale and two weaker features at $860 \mathrm{~cm}^{-1}$, due to the $\mathrm{C}=\mathrm{S}$ stretch (also referred to as the symmetric stretch by Hudgins et al. 1993), and at $520 \mathrm{~cm}^{-1}$, due to the $\mathrm{O}-\mathrm{C}-\mathrm{S}$ bend. The solid-phase peak position of the main band is shifted to lower frequency with respect to the gas-phase value, indicating that the overall interaction among OCS molecules in the solid phase is attractive. On warm-up from $10 \mathrm{~K}$ peak position of the main band shifts to lower frequencies; the FWHM decreases up to $50 \mathrm{~K}$ and then, between 50 and $60 \mathrm{~K}$, increases while a change in the profile occurs. This indicates a phase transition in the ice structure (Hudgins et al. 1993) (see Fig. 2).

The spectral properties of OCS strongly depend on the host molecules it is mixed in with (Fig. 3). Several ice mixtures have been studied (Hudgins et al. 1993; this work). Figure 2 shows the FWHM (measured on an intensity scale) against the peak positions as a function of mixture and temperature. No simple relationships have been found between peak position or FWHM of the OCS band and dipole moment or polarizability of the embedding matrix molecules, with bond size or with size of the site. For example, the spread in FWHM of the OCS band is as large as $10 \mathrm{~cm}^{-1}$ for OCS in various mixtures with nonpolar species. Likewise, OCS in mixtures with molecules with very disparate dipole moments can still have very similar FWHM. The general trend observed is similar to that reported for $\mathrm{CO}$ by Sandford et al. (1988). However, unlike CO, peak position and width of the OCS band in mixture with water depend on the relative amounts of the two species (Fig. 4). This behavior has also been observed for $\mathrm{CO}_{2}$ (Sandford \& Allamandola 1990).

The profile (shape, width, and position) of the OCS band in mixtures with water depends on the $\mathrm{H}_{2} \mathrm{O} / \mathrm{OCS}$ ratio (Fig. 4) and on the temperature. The band gets narrower and shifts to higher frequencies as the temperature increases. Up to about $80 \mathrm{~K}$ (i.e., at the sublimation temperature of pure OCS) this behavior can be ascribed to annealing effects of the $\mathrm{H}_{2} \mathrm{O}$

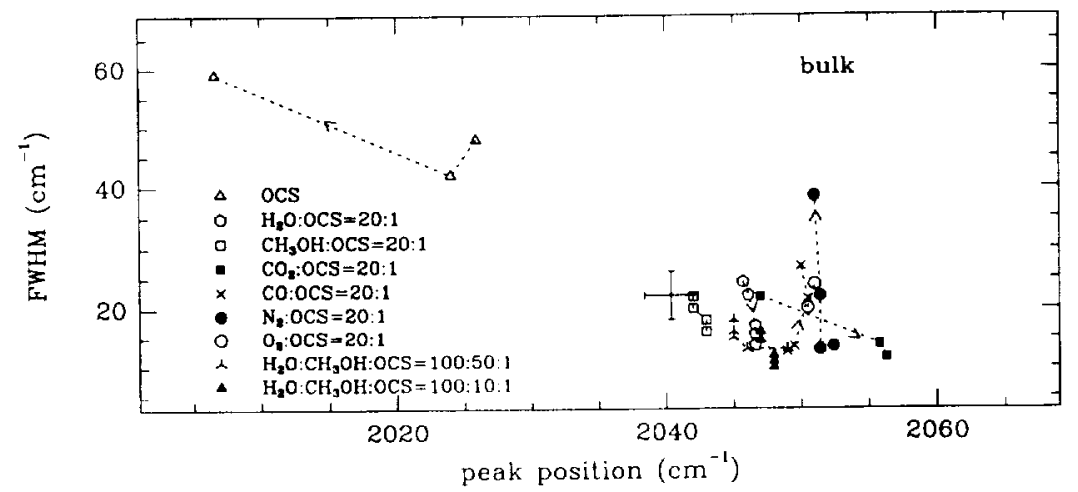

FIG 2-FWHM (measured on an intensity scale) is plotted vs. peak position of the main OCS band for OCS frozen in various mixtures (Hudgins et al. 1993 ; this

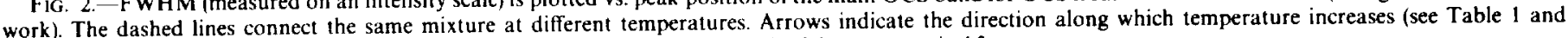
Hudgins et al. for temperature values). The cross indicates peak position and width of the astronomical feature. 
TABLE 1

Position and Width of the Main OCS Band in Various ICe Mixtures at DifFerent TeMPERATURes (This Work)

\begin{tabular}{|c|c|c|c|}
\hline Ice Composition & $\begin{array}{c}T \\
(\mathbf{K})\end{array}$ & $\begin{array}{c}\text { Peak } \\
\text { Position } \\
\left(\mathrm{cm}^{-1}\right)\end{array}$ & $\begin{array}{c}\text { FWHM } \\
\left(\mathrm{cm}^{-1}\right)\end{array}$ \\
\hline $\mathrm{H}_{2} \mathrm{O}: \mathrm{OCS}=30: 1 \ldots \ldots \ldots \ldots \ldots \ldots$ & $\begin{array}{r}10 \\
40 \\
80 \\
120 \\
140\end{array}$ & $\begin{array}{l}2048 \\
2048 \\
2048 \\
2048 \\
2048\end{array}$ & $\begin{array}{l}23 \\
22 \\
17 \\
14.5 \\
12.5\end{array}$ \\
\hline $\mathrm{H}_{2} \mathrm{O}: \mathrm{OCS}=250: 1 \ldots \ldots \ldots \ldots \ldots \ldots$ & $\begin{array}{r}10 \\
40 \\
80 \\
120 \\
140\end{array}$ & $\begin{array}{l}2048 \\
2048 \\
2049 \\
2049 \\
2049\end{array}$ & $\begin{array}{c}14.5 \\
12.5 \\
9 \\
8.5 \\
7.5\end{array}$ \\
\hline $\mathrm{CH}_{3} \mathrm{OH}: \mathrm{OCS}=10: 1 \ldots \ldots \ldots \ldots \ldots$ & $\begin{array}{r}10 \\
40 \\
80 \\
100\end{array}$ & $\begin{array}{l}2040 \\
2040 \\
2041 \\
2041\end{array}$ & $\begin{array}{l}27 \\
25 \\
22 \\
22\end{array}$ \\
\hline $\mathrm{CH}_{3} \mathrm{OH}: \mathrm{OCS}=20: 1 \ldots \ldots \ldots \ldots \ldots \ldots$ & $\begin{array}{r}10 \\
40 \\
80 \\
100\end{array}$ & $\begin{array}{l}2042 \\
2042 \\
2043 \\
2043\end{array}$ & $\begin{array}{l}22 \\
20 \\
18 \\
16\end{array}$ \\
\hline $\mathrm{H}_{2} \mathrm{O}: \mathrm{CH}_{3} \mathrm{OH}: \mathrm{OCS}=3: 10: 1 \ldots \ldots \ldots$ & $\begin{array}{r}10 \\
40 \\
80 \\
100 \\
120\end{array}$ & $\begin{array}{l}2043 \\
2042 \\
2043 \\
2043 \\
2045\end{array}$ & $\begin{array}{l}26 \\
24 \\
21 \\
20 \\
18\end{array}$ \\
\hline $\mathrm{H}_{2} \mathrm{O}: \mathrm{CH}_{3} \mathrm{OH}: \mathrm{OCS}=10: 5: 1 \ldots \ldots \ldots$ & $\begin{array}{l}10 \\
40 \\
80\end{array}$ & $\begin{array}{l}2047 \\
2046 \\
2047\end{array}$ & $\begin{array}{l}25 \\
22.5 \\
20.5\end{array}$ \\
\hline $\mathrm{H}_{2} \mathrm{O}: \mathrm{CH}_{3} \mathrm{OH}: \mathrm{OCS}=100: 50: 1 \ldots \ldots$ & $\begin{array}{r}10 \\
40 \\
80 \\
100 \\
120\end{array}$ & $\begin{array}{l}2045 \\
2045 \\
2045 \\
2046 \\
2049\end{array}$ & $\begin{array}{l}18 \\
16 \\
15 \\
13 \\
13\end{array}$ \\
\hline $\mathrm{H}_{2} \mathrm{O}: \mathrm{CH}_{3} \mathrm{OH}: \mathrm{OCS}=100: 10: 1 \ldots \ldots$ & $\begin{array}{r}10 \\
40 \\
80 \\
100 \\
120\end{array}$ & $\begin{array}{l}2048 \\
2047 \\
2048 \\
2048 \\
2048\end{array}$ & $\begin{array}{l}16 \\
14.5 \\
12 \\
11 \\
10\end{array}$ \\
\hline
\end{tabular}

matrix. This annealing will continue at higher temperatures. However, OCS sublimates above $80 \mathrm{~K}$, and changes in the OCS band profile at these temperatures are likely related to the increasing $\mathrm{H}_{2} \mathrm{O} / \mathrm{OCS}$ ratio. An increasing $\mathrm{H}_{2} \mathrm{O} / \mathrm{OCS}$ ratio shifts the band to higher frequencies and leads to narrower profiles (Fig. 4). Also, we observed a decrease in the band area indicating OCS loss. Similar behavior has been observed in $\mathrm{CH}_{3} \mathrm{OH}$ :OCS and $\mathrm{CO}_{2}$ : OCS mixture (i.e., in mixtures with

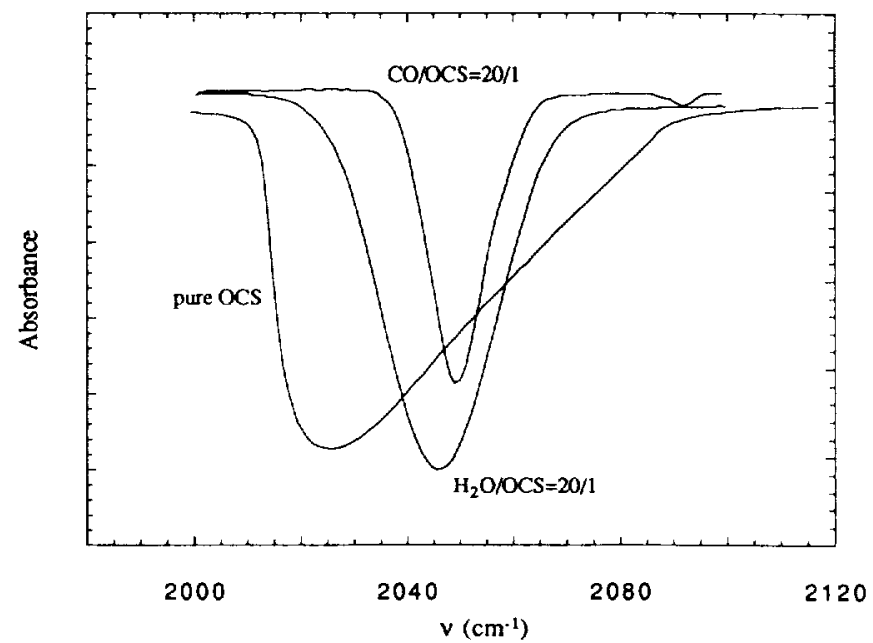

FIG. 3.- IR absorption spectra of solid OCS $(10 \mathrm{~K})$ pure and mixed with $\mathrm{CO}$ and $\mathrm{H}_{2} \mathrm{O}$. Spectra have been plotted on an arbitrary scale since here we are interested in a comparison of band profiles.

species with sublimation temperature higher than OCS). In contrast, the FWHM increases and the peak position remains almost constant in mixtures $(20: 1)$ with $\mathrm{CO}, \mathrm{N}_{2}$, and $\mathrm{O}_{2}$ (i.e., species with sublimation temperature lower then OCS). The $2340 \mathrm{~cm}^{-1} \mathrm{CO}_{2}$ band in mixtures of $\mathrm{H}_{2} \mathrm{O}: \mathrm{CO}_{2}(50: 1,20: 2$, and $5: 1$ ) shows similar behavior with temperature (Sanford $\&$ Allamandola 1990). In mixtures $\mathrm{H}_{2} \mathrm{O}: \mathrm{CH}_{3} \mathrm{OH}$ : OCS the peak position and width of the OCS band range between values assumed in $\mathrm{H}_{2} \mathrm{O}$ : OCS and $\mathrm{CH}_{3} \mathrm{OH}$ :OCS mixtures, depending on the $\mathrm{H}_{2} \mathrm{O} / \mathrm{CH}_{3} \mathrm{OH}$ ratio (Table 1).

In summary, the position of the main OCS band in different mixtures is close to the position of the absorption band observed in interstellar ices at $2041 \mathrm{~cm}^{-1}(4.9 \mu \mathrm{m})$. However, laboratory data show that the exact profile (position, width, and shape) of the OCS band depends on the host molecule and on the temperature of the sample, and no simple relationships exist among these parameters.

\section{MiE CALCUlations}

Particle size and shape can have very important effects on the profile of absorption features. For strong transitions in small particles, this class of electromagnetic modes is referred to as "surface modes" (van de Hulst 1957; Bohren \& Huffman 1983). Because of surface modes, absorption features can be shifted with respect to laboratory (bulk) spectra, and subpeaks appear. These effects can be easily understood in the Rayleigh

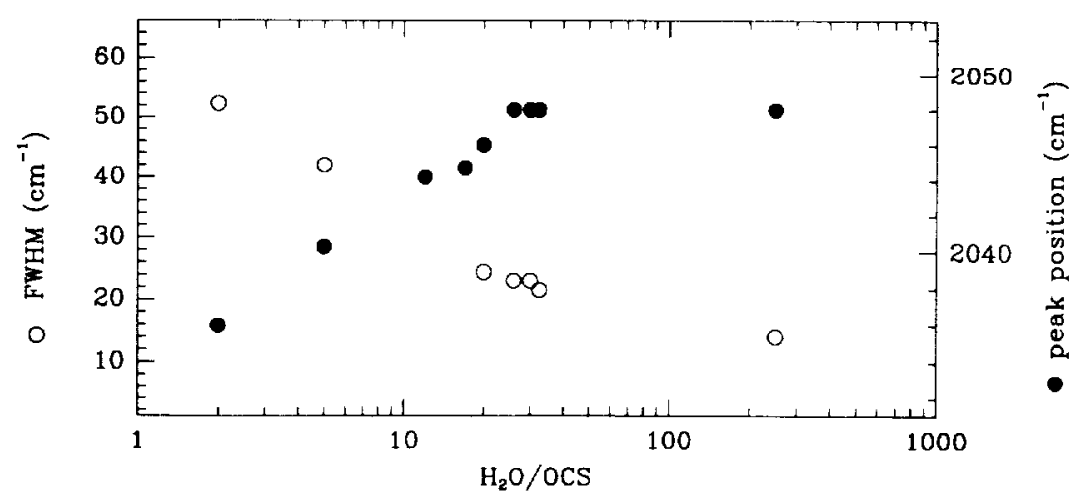

FIG. 4.-Peak position and FWHM in laboratory spectra of binary mixtures of $\mathrm{H}_{2} \mathrm{O}$ and $\mathrm{OCS}$, at $10 \mathrm{~K}$, are plotted vs. $\mathrm{H}_{2} \mathrm{O} / \mathrm{OCS}$ ratio 


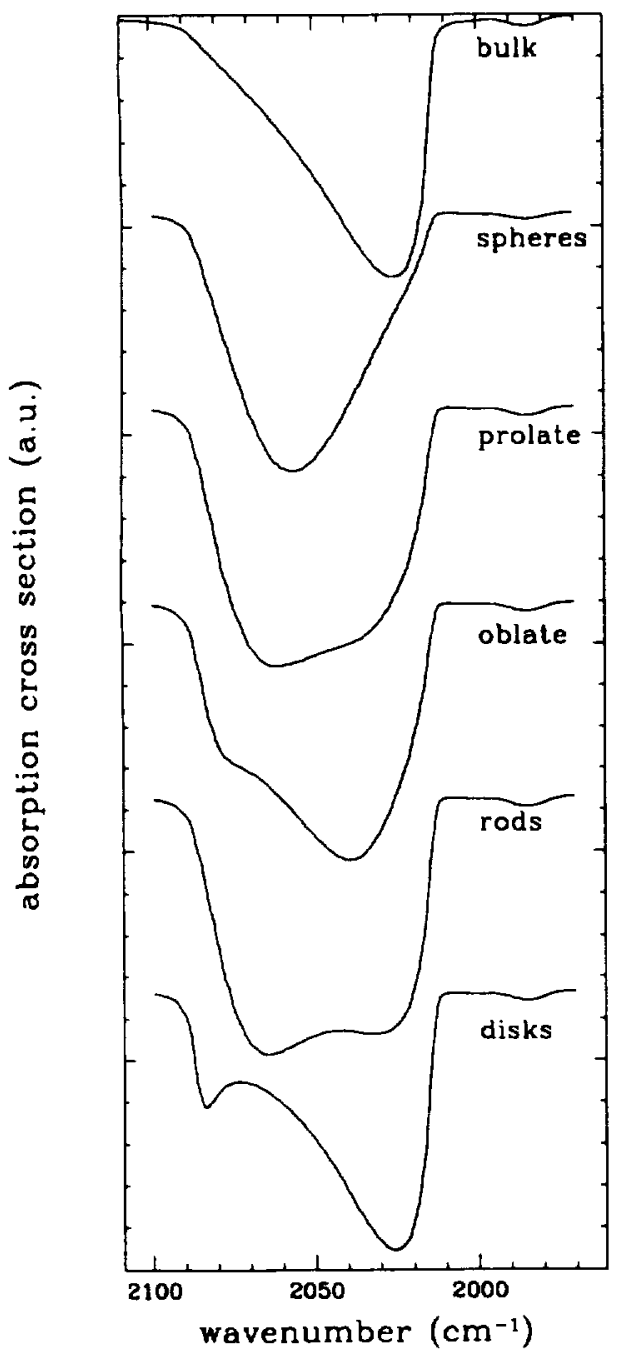

Fig. 5. - Calculated absorption cross sections in the Rayleigh limit for various homogeneous spheroids consisting of pure OCS plotted in arbitrary units (a.u.). The prolate and oblate spheroids have axial ratios of 0.2. The upper feature is the laboratory spectrum of pure OCS at $10 \mathrm{~K}$. limit $(2 \pi a \ll \lambda, a$ being the radius of the particle and $\lambda$ the wavelength of the incident photon) since the particle may be considered placed in a homogeneous electric field and electrostatics applies. Because of its electric polarization, the applied homogeneous electric field induces a dipole in the particle the strength of which depends on the dielectric constant $\left(\epsilon=\epsilon_{1}\right.$ $\left.+i \epsilon_{2}\right)$ of the particle as well as its shape. The field outside the particle is then a superposition of the uniform applied field and the particle's dipole field, while inside the particle the field is homogeneous. In the spectral range of an absorption feature, where $\epsilon$ varies considerably and its real part may even be negative, the internal field of the particle can be very large (Landau \& Lifshitz 1960). In these instances an oscillator inside the particle is strongly affected by this field and the spectral feature of a small particle can be shifted with respect to the bulk material from about the maximum of $\epsilon_{2}$ to frequencies where $\epsilon_{1}$ is small or negative. In addition to frequency shifts obtained for spherical grains, spheroidal grains give rise to spectral structure (subpeaks) not present in the laboratory spectra. We have performed Mie scattering calculations for spheroidal particles in the Rayleigh limit for various mixtures containing OCS using the optical constants measured by Hudgins et al. (1993). The results are summarized in Figures 5 and 6.

Figure 5 compares the laboratory spectrum of pure OCS ice at $10 \mathrm{~K}$ with Mie-calculated absorption cross sections for small particles. The general points discussed above are quite apparent in this figure. The peak position shifts from the bulk position, $2025 \mathrm{~cm}^{-1}$, to about $2060 \mathrm{~cm}^{-1}$ for spheres. For spheroidal particles, where more than one resonance occurs, these subpeaks merge into one broad feature.

The same calculations and comparisons have been performed with mixtures containing OCS (namely, $\mathrm{H}_{2} \mathrm{O}: \mathrm{OCS}=$ 20:1 and $\mathrm{H}_{2} \mathrm{O}: \mathrm{OCS}=2: 1$ ), and the results are summarized in Figure 6, where the FWHM of the calculated absorption is plotted against peak position. As a consequence of surface modes, the peak position is shifted to higher frequencies (shorter wavelengths) with respect to laboratory data. In the case of oblate particles, the peak position ranges between the bulk position and the position calculated for spheres, with disks closest to the bulk position. In the case of prolate par-

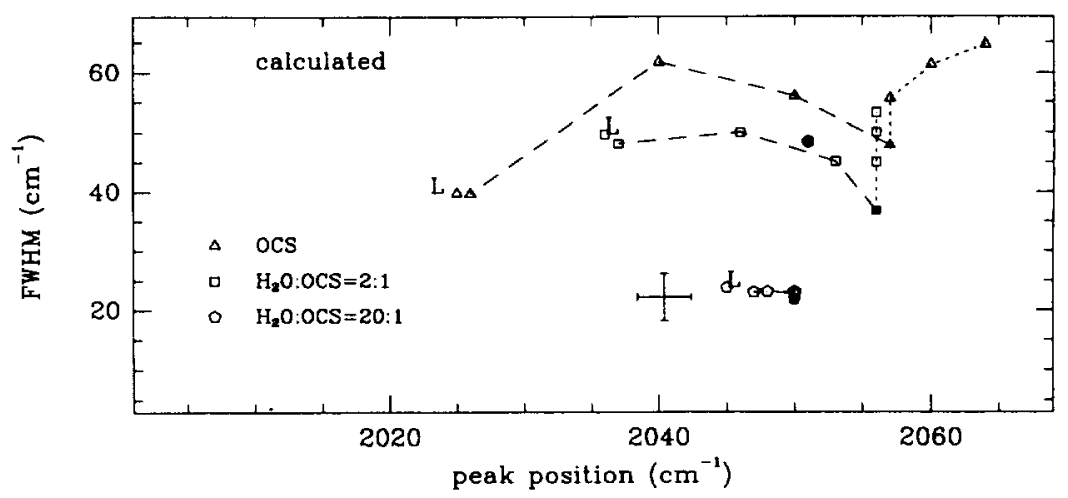

FIG. 6.-FWHM (measured on an absorbance scale) vs. peak position of the main OCS band for laboratory and calculated spectra: triangles, pure OCS ice at

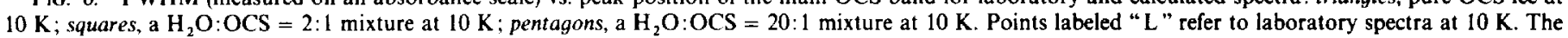

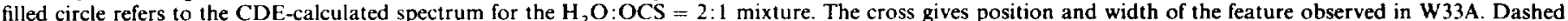

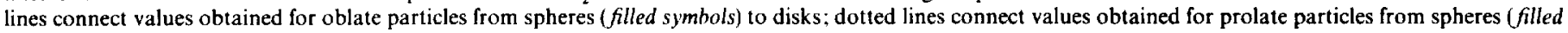
symbols) to rods. 
ticles, the peak position also shifts to higher frequencies with respect to spheres for pure OCS ice. But for $\mathrm{H}_{2} \mathrm{O} / \mathrm{OCS}>20$ mixtures, the peak position hardly shifts. These calculations have shown that particle size and shape effects are important only for high concentrations of OCS. Thus, when OCS is a minor component in the mixture, the contribution to the induced electric field due to $\epsilon(\mathrm{OCS})$ is very small and the effects are negligible. In $\mathrm{H}_{2} \mathrm{O}$-rich ices (OCS concentration less than 0.05 ), the OCS band is weak and hence the peak position and profile are hardly sensitive to the shape of the particle. This conclusion is general and holds for other diluted OCS mixtures as well. Taking the difference in intrinsic strength into account, this result is in good agreement with those for diluted $\mathrm{CO}$ mixtures (Tielens et al. 1991). In general, the FWHM increases from spheres to rods through prolate particles due to the merging of the subpeaks. Similar trends have been obtained for various mixtures containing $C O$ (Tielens et al. 1991).

Finally, shape and size distribution tend to smooth the spectral structures, and the only effect remaining, as compared to laboratory data, is a shift in the peak position and a broadening of the feature. The results obtained for a continuous distribution of ellipsoidal shapes (CDEs; Bohren \& Huffman 1983) illustrate this point (Fig. 6).

\section{SOLID OCS IN W33A: ASTROPHYSICAL IMPLICATIONS}

In view of the laboratory experiments discussed in this paper, we propose that the $2041 \mathrm{~cm}^{-1}$ band observed toward W33A is due to solid OCS embedded in icy grain mantles. We have compared the observed $2041 \mathrm{~cm}^{-1}$ band with laboratory and theoretical spectra of various mixtures. However, we have been mainly interested in comparison with astrophysically relevant mixtures with molecules either observationally known or theoretically expected to be abundant ice mantle components (Tielens \& Allamandola 1987a, b; Tielens 1989).

Comparison of peak position and width of the OCS band in different laboratory mixtures with observed values (Fig. 2) show that solid OCS in W33A is not embedded in a nonpolar matrix. Mie calculations show that when OCS has a high concentration (i.e., for OCS concentrations above 0.05 ) the profile is affected by surface modes and subpeaks occur (Figs. 5 and 6). These are not observed, and hence we can conclude that the OCS concentration in icy grain mantles toward W33A is less than 0.05 .

Thus OCS is only a minor component (concentration less than 0.05 ) in the interstellar grain mantles toward W33A. Binary mixtures of $\mathrm{H}_{2} \mathrm{O}$ with traces of OCS can provide good fits to either peak position or width but not both. Adding methanol to the mixtures improves the fit.

Laboratory spectra are compared to the observations in Figures $7 a$ and $7 b$. In view of the discussion above, we have limited ourselves in this detailed comparison to mixtures containing methanol. The fit improves, particularly on the shortwavelength side of the feature, when the $\mathrm{CH}_{3} \mathrm{OH}$ abundance increases relative to that of $\mathrm{H}_{2} \mathrm{O}$. Good results are obtained with laboratory mixtures rich in $\mathrm{CH}_{3} \mathrm{OH}$ (namely, $\mathrm{CH}_{3} \mathrm{OH}: \mathrm{OCS}=20: 1 \quad$ and $\quad \mathrm{H}_{2} \mathrm{O}: \mathrm{CH}_{3} \mathrm{OH}: \mathrm{OCS}=3: 10: 1$ mixtures). This leads to the conclusion that OCS is mainly embedded in mantles with little or no water and rich in methanol. However, these grains can only be a minor component along the line of sight in view of the observed $\mathrm{OCS} / \mathrm{H}_{2} \mathrm{O}$ $\left(4 \times 10^{-4}\right.$; see below) and $\mathrm{CH}_{3} \mathrm{OH} / \mathrm{H}_{2} \mathrm{O}(0.05-0.5$; Grim et al. 1991; Tielens \& Allamandola 1987b) ratios. The presence of (almost) pure methanol grain mantles is in agreement with the analysis of the IR spectrum of the protostar GL 2136 (Skinner et al. 1992). For this source, the profile of the 8.9 and $9.7 \mu \mathrm{m}$ $\mathrm{CH}_{3} \mathrm{OH}$ bands also implied the presence of methanol-rich $\left(\mathrm{CH}_{3} \mathrm{OH} / \mathrm{H}_{2} \mathrm{O}>0.5\right)$ interstellar ice mantles despite the observed low column density ratio of $\mathrm{CH}_{3} \mathrm{OH}$ to $\mathrm{H}_{2} \mathrm{O}(0.05)$. Such a differentiation of grain mantles along the same line of sight has also been inferred from the shape and peak position of the solid $\mathrm{CO}$ band toward a large variety of objects (Tielens et al. 1991). Hence, chemical differentiation seems to be a general characteristic of interstellar ice mantles and may reflect the importance of a variety of chemical or physical (i.e., thermal outgassing) processes in molecular clouds.

OCS is a common gas-phase molecule in the ISM, present in both quiescent molecular clouds and regions of star formation. Typically, its abundance with respect to hydrogen is $N_{\text {gas }}(\mathrm{OCS}) / N_{\text {zas }}\left(\mathrm{H}_{2}\right)=10^{-8}$ to $2 \times 10^{-9}$ (Irvine, Goldsmith, \& Hjalmarson 1987; Ohishi, Irvine, \& Kaifu 1992). Using laboratory values for the intrinsic band strength the column density of solid OCS toward W33A is $2 \times 10^{16} \mathrm{~cm}^{-2}$. Gaseous $\mathrm{H}_{2}$ and solid $\mathrm{H}_{2} \mathrm{O}$ column densities toward $\mathrm{W} 33 \mathrm{~A}$ have been derived by Tielens et al. (1991). The column density of solid OCS translates into abundances of solid OCS with respect to gaseous $\mathrm{H}_{2}$ and solid $\mathrm{H}_{2} \mathrm{O}$ of $X_{\text {Ocs }}=N_{\text {solid }}(\mathrm{OCS}) / N_{\text {aas }}\left(\mathrm{H}_{2}\right)=7 \times 10^{-8}$ and $N_{\text {solid }}(\mathrm{OCS}) / N_{\text {solid }}\left(\mathrm{H}_{2} \mathrm{O}\right)=4 \times 10^{-4}$. Hence, solid OCS is more abundant in this line of sight than gas-phase OCS, in general. At present, it is not known whether this is a general property of solid OCS. A number of different chemical routes for the formation of solid OCS have been proposed. This includes direct freeze-out from the gas phase. However, the high measured solid OCS abundance in this line of sight may argue against this. Grain surface reactions of atomic $S$ with $\mathrm{CO}$ and atomic $\mathrm{O}$ with $\mathrm{CS}$, analogous to the reaction $\mathrm{O}+\mathrm{CO} \rightarrow \mathrm{CO}_{2}$ have also been proposed (Tielens \& Allamandola 1987b). Both reactions are known to occur at $10 \mathrm{~K}$ in solid matrices. Calculations show that $\mathrm{OCS} / \mathrm{H}_{2} \mathrm{O}$ ratios up to $5 \times 10^{-3}$ can be obtained this way. The predominance of OCS in $\mathrm{CH}_{3} \mathrm{OH}$-rich mixtures is quite interesting. Methanol is thought to be formed through $\mathrm{H}$-addition reactions to $\mathrm{CO}$ (Tielens \& Allamandola 1987a). In this picture, one might thus expect high abundances of the molecule $\mathrm{CH}_{3} \mathrm{SH}$. Moreover, $\mathrm{CO}_{2}$ might also be expected to be predominantly present in a $\mathrm{CH}_{3} \mathrm{OH}$-rich environment. Finally, OCS may also be formed by $U V$ and/or ion irradiation of mixtures containing $\mathrm{CO}$ and sulfur-bearing compounds, notably $\mathrm{H}_{2} \mathrm{~S}$. Again, analogous systems lead to $\mathrm{CO}_{2}$ formation. However, the efficiency of this process in interstellar space is not known. It is clear from this discussion that a comparison with solid $\mathrm{CO}_{2}$ abundances (possible after the launch of the Infrared Space Observatory [ISO]) may be very fruitful in elucidating the reaction pathway involved in $\mathrm{OCS}$ (and $\mathrm{CO}_{2}$ ) formation. Further, a more extensive observational study of interstellar OCS will also be useful.

Other carriers have been suggested for the $2041 \mathrm{~cm}^{-1}(4.9$ $\mu \mathrm{m})$ feature observed toward W33A (Geballe et al. 1985; Larson et al. 1985; Grim et al. 1991). In particular, a possible candidate is the overtone of the $\mathrm{C}-\mathrm{O}$ stretching $\left(2 v_{2}\right)$ mode in methanol. Indirect support for this identification is provided by the various other modes of solid methanol that have been identified in protostellar spectra, including the $\mathrm{C}-\mathrm{O}$ stretch and the $\mathrm{CH}_{3}$ and $\mathrm{OH}$ stretching, deformation, and rocking modes (Tielens \& Allamandola 1987b; Grim et al. 1991; Allamandola et al. 1992; Skinner et al. 1992). However, comparisons between laboratory and observed spectra show that the $2 v_{2}$ mode, both in pure methanol and in mixture with water 


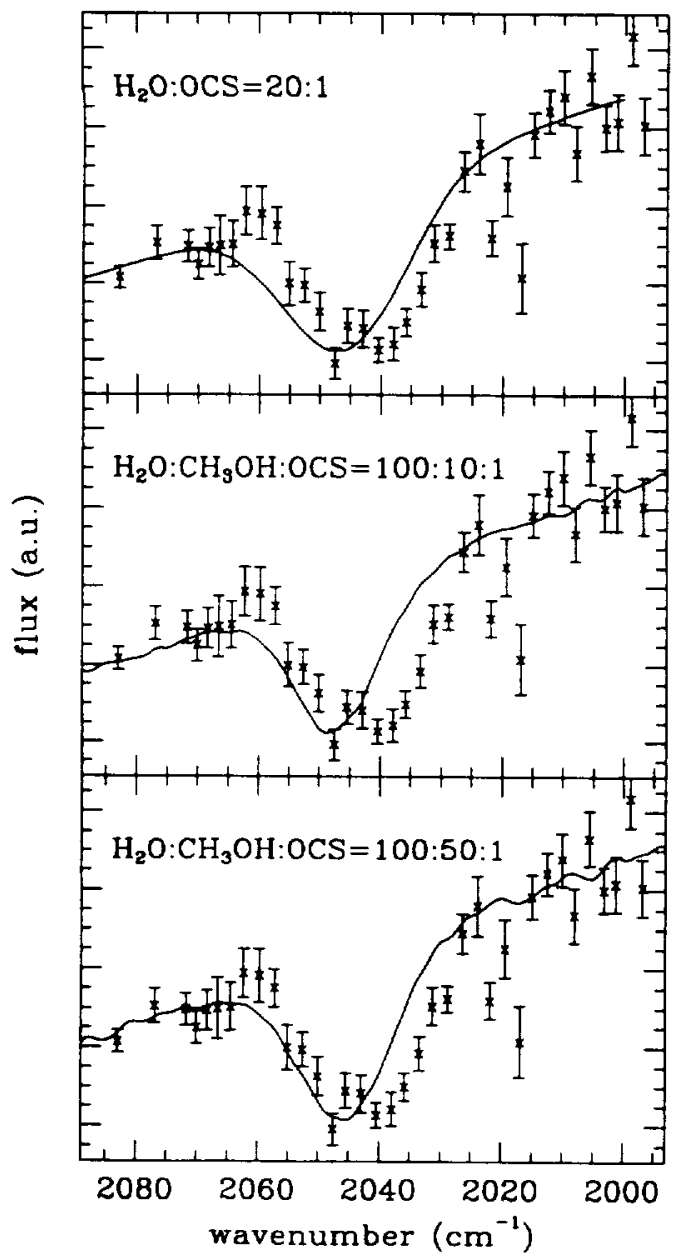

Fig. $7 a$

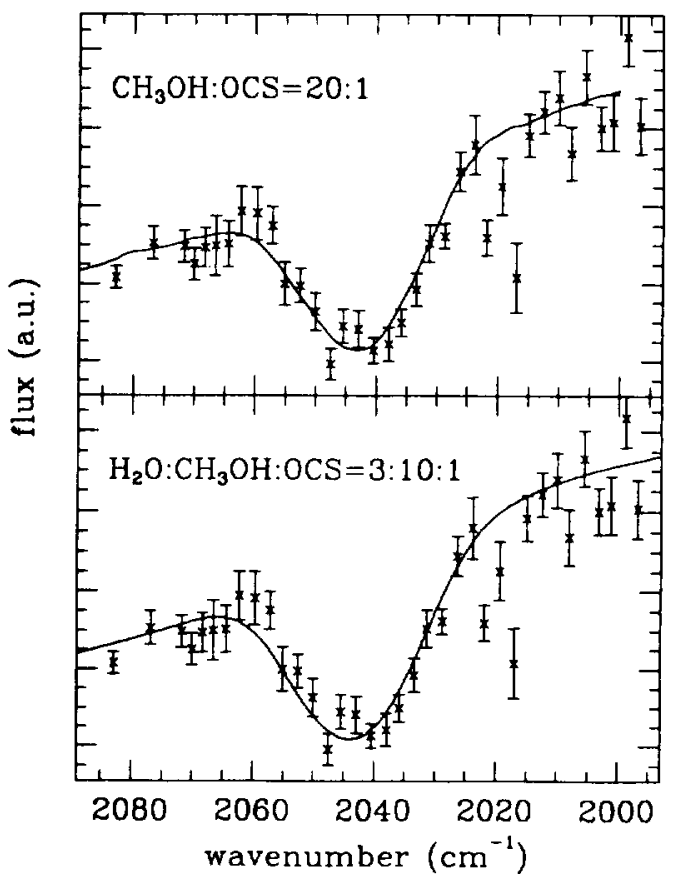

FIG. $7 b$

FIG. 7.-Comparisons of the observed $4.9 \mu \mathrm{m}$ band in W33A with laboratory spectra

$\left(\mathrm{H}_{2} \mathrm{O}: \mathrm{CH}_{3} \mathrm{OH}=1: 1\right)$, does not provide a good fit since the band in the laboratory spectra is broader than the observed one (Fig. 8; Grim et al. 1991). Furthermore, the $2 v_{2}$-mode band was not observed in a mixture $\mathrm{H}_{2} \mathrm{O}: \mathrm{CH}_{3} \mathrm{OH}=10: 1$, which indicates that in a water-rich environment the strength of this band is reduced. Finally, assuming that the $2041 \mathrm{~cm}^{-1}$ band is due to the $2 v_{2}$ mode in methanol, the optical depths of other observed bands attributed to methanol would be 7.5 and 5 in

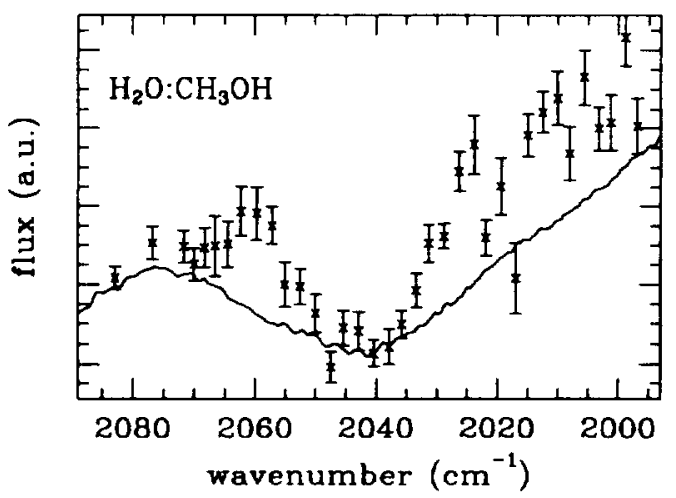

Fig. 8. - Comparison of the observed $4.9 \mu \mathrm{m}$ band in W33A with the overtone of the methanol $\mathrm{C}-\mathrm{O}$ stretch in a $\mathrm{H}_{2} \mathrm{O}: \mathrm{CH}_{3} \mathrm{OH}=1: 1$ mixture. First, the laboratory spectrum has been normalized to the continuum, and then the baseline, defined in the text, has been used for the comparison. the case of the $2825 \mathrm{~cm}^{-1}(3.53 \mu \mathrm{m})$ band and the $1460 \mathrm{~cm}^{-1}$ $(6.8 \mu \mathrm{m})$ band, respectively. These values are much larger than observed (0.39 and 0.7; Allamandola et al. 1992; Tielens \& Allamandola 1987b).

\section{CONCLUSIONS}

We have observed the $2041 \mathrm{~cm}^{-1}(4.9 \mu \mathrm{m})$ band in the protostar W33A. We attribute this band to solid OCS in interstellar ice mantles along the line of sight. We have studied this OCS band in a variety of mixtures in the laboratory. The peak position and shape of this band are sensitive to the molecular environment of OCS. Good fits to the observed interstellar spectrum are obtained for mixtures rich in methanol. This leads to the conclusion that OCS is mainly embedded in mantles with little or no water and rich in methanol. However, these grains can only be a minor component along the line of sight in view of the observed $\mathrm{OCS} / \mathrm{H}_{2} \mathrm{O}$ and $\mathrm{CH}_{3} \mathrm{OH} / \mathrm{H}_{2} \mathrm{O}$ ratios. This requires chemical differentiation of grain mantles along the line of sight. In order to confirm this conclusion and to better understand the composition of interstellar icy grain mantles, more experimental and observational work is necessary. It is important to search for the $2041 \mathrm{~cm}^{-1}$ feature in a large sample of sources including both field stars and embedded objects in different stages of their pre-main-sequence evolution, thus sampling a variety of physical conditions. 
Comparison of the OCS abundances with those of solid $\mathrm{H}_{2} \mathrm{O}$, $\mathrm{CH}_{3} \mathrm{OH}, \mathrm{CO}$, and $\mathrm{XCN}$ will help to determine the importance of various proposed OCS formation routes; likewise, the profile of the OCS band reveals much about the composition of the mixture it is embedded in. Furthermore, OCS is very similar, in its physical and chemical properties, to $\mathrm{CO}_{2}$. The latter cannot be observed at present because of telluric absorption, but this will change after the launch of ISO. The comparison between solid OCS and $\mathrm{CO}_{2}$ can greatly elucidate grain surface chemistry. Finally, the effects of UV and ion irradiation on icy samples containing sulfur-bearing compounds are to be studied.

We wish to thank $M$. Bernestein and $R$. Bohn for their invaluable assistance throughout the experimental work at NASA/Ames and G. Strazzulla for helpful discussions about the paper. M. E. P. acknowledges the financial support of the Italian Space Agency (ASI).
Allamandola, L. J, Sanford S A. Tielens, A G. G. M \& Herbst, T. M. 1992 ApJ, 399, 134

Allamandola, L. J., Sandford, S. A., \& Valero, G. J. 1988, Icarus, 76, 225

Baratta, G. A., \& Strazzulla, G. 1990, A\&A, 240, 429

Bohren, C. F. \& Huffman, D. R. 1983, Absorption and Scattering of Light by Small Particles (New York: Wiley)

Brown, P. D., Charnley, S. B., \& Millar, T. J. 1988, MNRAS, 231, 409

d'Hendecourt, L. B., Allamandola, L. J., \& Greenberg. J. M. 1985, A\&A, 152, 130

Gebelle, T R Baas, F Greenberg J M \& Schutte, W 1985, A\&A, 146, L6

Greenberg, J. M. 1982, in Comets, ed. L. J. Wilkening (Tucson: Univ. Arizona Press), 131

Grim, R. J. M., Baas, F., Geballe, T. R., Greenberg, J. M., \& Schutte, W. 1991, A\&A, 243, 473

Grim, R. J. M., \& Greenberg, J. M. 1987, ApJ, 321, L91

Hudgins, D M Sanford, S. A Allamandola, L. J. \& Tielens, A. G. G. M 1993, ApJS, 86, 713

Irvine, W. M., Goldsmith, P. F., \& Hjalmarson, A. 1987, in Interstellar Processes, ed. D. J. Hollenbach \& H. A. Thronson (Dordrecht : Reidel), 561

Lacy, J. H., Baas, F., Allamandola, L. J., Persson, S. E., McGregor, P. J., Lonsdale, C. J. Geballe, T. R., \& van de Bult, C. E. P. 1984, ApJ, 276, 533

Landau, L. D., \& Lifshitz, E. M. 1960, Electrodynamics of Continuous Media (New York: Pergamon)

Larson, H. P., Davis, D. S., Black, J. H., \& Fink, U. 1985, ApJ, 299, 873

Ohishi, M., Irvine, W. M., \& Kaifu, N. 1992, in Astrochemistry of Cosmic Phenomena, ed. P. D. Singh (Dordrecht : Kluwer), 171
Palumbo, M. E \& Strazzulla, G. 1993, A\&A, 269,568

Stanford, S. A., \& Allamandola, L. J. 1990, ApJ, 355, 357

Stanford, S. A., Allamandola, L. J., Tielens, A. G. G. M., \& Valero, G. J. 1988 ApJ, 329, 498

Skinner, C. J., Tielens, A. G. G. M., Barlow, M. J., \& Justtanont, K. 1992, ApJ, $399, \mathrm{~L} 79$

Strazzulta, G. \& Baratta, G. A. 1991, A\& A, 241, 310

Tielens, A. G. G. M. 1989, in IAU Symp. 135, Interstellar Dust, ed. L. J. Allamandola \& A. G. G. M. Tielens (Dordrecht: Reidel), 239

Tielens, A. G. G. M., \& Allamandola, L. J. 1987a, in Interstellar Processes, ed D. J. Hollenbach \& H. A. Thronson (Dordrecht ; Reidel), 397 1987 b, in Physical Processes in Interstellar Clouds, ed. G. E. Morfill \& M. Schoeler (Dordrecht: Reidel), 333

Tielens, A. G. G. M., Allamandola, L. J., Bregman, J., Goebel, J., d'Hendecourt L. B., \& Witteborn, F. C. 1984, ApJ, 287,697

Tielens, A. G. G. M., \& Hagen, W. 1982, A\&A, 114, 245

Tielens, A. G. G. M., Tokunaga, A. T., Geballe, T. R., \& Baas, F. 1991, ApJ, 381,181

Tokunaga, A. T., Smith, R. G., \& Irwin, E. 1987, in Infrared Astronomy with Arrays, ed. C. G. Wynn-Williams \& Becklin (Honolulu: Univ. of Hawaii, Inst. Astron.J, 367

van de Hulst. H. C. 1957, Light Scattering by Small Particles (New York: Dover) 